BRX TH-452

HUB-EP-99/11

hep-th/9902210

\title{
Localized intersections of M5-branes and four-dimensional superconformal field theories
}

\author{
August 20, 2018 \\ ${ }^{1}$ Martin Fisher School of Physics \\ Brandeis University \\ Waltham, MA 02138, U.S.A. \\ ${ }^{2}$ Institut für Physik \\ Humboldt-Universität \\ D-10115 Berlin \\ Germany
}

Ansar Fayyazuddin ${ }^{1 *}$ and Douglas J. Smith ${ }^{2 \dagger}$

\begin{abstract}
We write supersymmetry preserving conditions for infinite M5branes intersecting on a $(3+1)$-dimensional space. In contrast to previously known solutions, these intersections are completely localized. We solve the equations for a particular class of configurations which in the near-horizon decoupling limit are dual to $N_{f}=2 N_{c}$ SeibergWitten superconformal field theories with gauge group $\mathrm{SU}(N)$ and generalisations to $\mathrm{SU}(N)^{n}$. We also discuss the relationship to D3branes in the presence of an $A_{k}$ singularity.
\end{abstract}

*email: fayyaz@binah.cc.brandeis.edu

†email: smith@physik.hu-berlin.de 


\section{Introduction}

A recent conjecture due to Maldacena [1] asserts that gauge theories realized as worldvolume theories of branes can be described equivalently as gravity theories in the near-horizon geometry due to these branes. A precise correspondence between problems in gauge theory and problems in gravity has been elucidated in [2, 3].

One interesting field of application is four-dimensional conformal field theories. Of particular importance is the $\mathcal{N}=4 \mathrm{SU}(N)$ system realized by parallel D3-branes in type IIB string theory. Its near-horizon geometry is $A d S_{5} \times S^{5}$. A number of other conformal field theories with less supersymmetry have been constructed by employing various techniques [4, 5, 6, 7, 8, 9, 10, 11, 12, 13, 14, 15. The near-horizon geometry due to the branes is of the form $A d S_{5} \times M$ where $M$ is a compact manifold. The isometries of the near-horizon geometry are realized as global symmetries of the world-volume gauge theory of the branes. In particular, the $A d S$ factor is a signature of conformal invariance of the world-volume gauge theory since its isometry group is the conformal group of the field theory living at its boundary.

In this paper we study $\mathcal{N}=2$ superconformal field theories. We first describe the conditions for supersymmetry-preserving configurations of 5branes in 11-dimensional supergravity. This will allow us to write down the general form of the metric describing 5-branes with worldvolume $\mathbb{R}^{4} \times$ $\Sigma$ preserving at least one quarter of the maximum supersymmetry. This corresponds to $\mathcal{N}=2$ supersymmetry in 4 dimensions. We will then show how a specific configuration of this form reduces to a Hanany-Witten brane configuration [16] in 10-dimensional type IIA [17], realising a 4-dimensional conformal field theory. This configuration is special in that it receives no non-perturbative corrections and so one can safely describe the system in type IIA. We are able to solve the equations only when the NS5-branes are smeared in one direction. This smearing is typical of known configurations of intersecting branes, [18, 19, 20] and references therein. See also [21, 22, 23] for recent work on localised intersections. However, our method allows, in principle, the construction of fully localised solutions. We will take the nearhorizon limit of this supergravity solution which should be the Maldacena dual of the conformal field theory. We show that the space is of the form $A d S_{5} \times M$ as expected. The compact manifold $M$ has the isometry group $\mathrm{SO}(3) \times \mathrm{U}(1)$ matching the R-symmetry group of the field theory, there is also an additional $\mathrm{U}(1)$ due to the smearing. This last $\mathrm{U}(1)$ should disappear in 
the fully localized solution.

In addition we write down explicitly the T-dual [24, 25] (see also 26, 27. 28 ) geometry in Type IIB. This geometry describes D3-branes in the presence of an $A_{1}$ ALE singularity. This parallels the recent discussions of D3-branes at conifold singularities [10, 12] which is T-dual to NS5- and D4-brane configurations [29, 30, 31] with $N=1$ supersymmetry in four dimensions.

\section{Preservation of supersymmetry}

In this section we will discuss the conditions on the metric and 4-form $F$ for a supersymmetry-preserving solution of 11-dimensional supergravity. It is known that a configuration preserving some supersymmetry will always be a solution of the classical equations of motion provided the Bianchi identity and equations of motion for $F$ are satisfied. So we will start by analysing the restrictions imposed by the requirement that we preserve (in our case one quarter) supersymmetry. This is essentially a BPS condition and so ensures that the equations of motion are satisfied. We will then supplement the resulting conditions with the additional constraints on $F$.

The SUSY variation of the gravitino Rarita-Schwinger field $\Psi_{M}$ is:

$$
\delta_{\epsilon} \Psi_{M}=\left(\partial_{M}+\frac{1}{4} \omega_{b M}^{a} \hat{\Gamma}_{a}^{b}+\frac{1}{144} \Gamma_{M}{ }^{N P Q R} F_{N P Q R}-\frac{1}{18} \Gamma^{P Q R} F_{M P Q R}\right) \epsilon
$$

In order to find supersymmetric solutions we should choose the metric and the 4-form $F$ so that:

$$
\delta_{\epsilon} \Psi_{M}=0
$$

for some $\epsilon$ where in our conventions:

$$
\hat{\Gamma}_{(10)} \epsilon \equiv \hat{\Gamma}_{0123456789} \epsilon
$$

We want a solution which corresponds to an M5-brane with worldvolume $\mathbb{R}^{4} \times \Sigma$ with $\Sigma$ being a 2-cycle holomorphically embedded in $\mathbb{C}^{2}$ with complex coordinates $(v, s)$. I.e. $\Sigma$ is specified as the zero of a holomorphic function $f(v, s)$. We can also consider several 5 -branes by taking $\Sigma$ to be a sum of disconnected holomorphic 2-cycles. The metric should be symmetric under $4 \mathrm{~d}$ Lorentz transformations and rotations in the three transverse directions. 
If we pick a complex structure in the $x_{4}, x_{5}, x_{6}, x_{7}$ directions and assume that the metric is Hermitian in this subspace, the most general metric consistent with the above symmetries is of the form:

$$
d s^{2}=H_{1} \eta_{\mu \nu} d x^{\mu} d x^{\nu}+2 G_{m \bar{n}} d z^{m} d z^{\bar{n}}+H_{2} \delta_{\alpha \beta} d x^{\alpha} d x^{\beta}
$$

where (middle) Greek indices $\mu, \nu$ etc. correspond to $M=0,1,2,3$ and (beginning) Greek indices $\alpha, \beta$ etc. correspond to $M=8,9,10$. The two complex variables are defined by $z^{v}=v=x^{4}+i x^{5}$ and $z^{s}=s=x^{6}+i x^{7}$. $H_{1}, H_{2}$ and $G_{m \bar{n}}$ are arbitrary functions of $v, \bar{v}, s, \bar{s}$ and $r=\sqrt{\delta_{\alpha \beta} x^{\alpha} x^{\beta}}$. For later convenience we will also define the rescaled complex metric:

$$
g_{m \bar{n}}=H_{1}^{-1} G_{m \bar{n}}
$$

In order to recover Minkowski space far from any sources we must have asymptotically:

$$
\begin{aligned}
H_{1} & \rightarrow 1 \\
H_{2} & \rightarrow 1 \\
G_{v \bar{v}} & \rightarrow \frac{1}{2} \\
G_{s \bar{s}} & \rightarrow \frac{1}{2} \\
G_{v \bar{s}} & \rightarrow 0 \\
G_{s \bar{v}} & \rightarrow 0
\end{aligned}
$$

We now wish to analyse the condition for preserving supersymmetry. For a 5-brane with worldvolume $\mathbb{R}^{4} \times \Sigma$, the number of supersymmetries preserved is given by the number of spinors $\epsilon$ which satisfy [32, 33, 34, 35]:

$$
\sqrt{\operatorname{det} h} d \sigma^{1} d \sigma^{2} \epsilon=\hat{\Gamma}_{0123} \Gamma_{m \bar{n}}\left(\partial_{1} Z^{m} \partial_{2} Z^{\bar{n}}-\partial_{1} Z^{m} \partial_{2} Z^{\bar{n}}\right) d \sigma^{1} d \sigma^{2} \epsilon
$$

where $h_{i j}$ is the pullback of the spacetime metric $G_{m \bar{n}}$ onto $\Sigma, \sigma^{i}$ are the two (real) worldvolume coordinates on $\Sigma$ and $Z^{m}\left(\sigma^{1}, \sigma^{2}\right)$ are the target space coordinates of $\Sigma$. Now on $\Sigma$ we have $f\left(Z^{v}, Z^{s}\right)=0$ so:

$$
\partial_{i} Z^{s}=-\partial_{v} f\left(\partial_{s} f\right)^{-1} \partial_{i} Z^{v}
$$

We can now evaluate the determinant of $h_{i j}=\partial_{(i} Z^{m} \partial_{j)} Z^{\bar{n}} G_{m \bar{n}}$ as:

$$
\sqrt{\operatorname{det} h}= \pm i\left(\partial_{[1} Z^{v} \partial_{2]} Z^{\bar{v}}\right)\left(G_{v \bar{v}}-\overline{\left(\frac{\partial_{v} f}{\partial_{s} f}\right)} G_{v \bar{s}}-\frac{\partial_{v} f}{\partial_{s} f} G_{s \bar{v}}+\left|\frac{\partial_{v} f}{\partial_{s} f}\right|^{2} G_{s \bar{s}}\right)
$$


so the equation for preservation of supersymmetry becomes:

$$
\begin{aligned}
& \hat{\Gamma}_{0123}\left(\Gamma_{v \bar{v}}-\overline{\left(\frac{\partial_{v} f}{\partial_{s} f}\right)} \Gamma_{v \bar{s}}-\frac{\partial_{v} f}{\partial_{s} f} \Gamma_{s \bar{v}}+\left|\frac{\partial_{v} f}{\partial_{s} f}\right|^{2} \Gamma_{s \bar{s}}\right) \epsilon \\
= & i c\left(G_{v \bar{v}}-\overline{\left(\frac{\partial_{v} f}{\partial_{s} f}\right)} G_{v \bar{s}}-\frac{\partial_{v} f}{\partial_{s} f} G_{s \bar{v}}+\left|\frac{\partial_{v} f}{\partial_{s} f}\right|^{2} G_{s \bar{s}}\right) \epsilon
\end{aligned}
$$

where $c= \pm 1$ corresponding to a brane or anti-brane. So we end up with the conditions that the spinor $\epsilon$ must satisfy:

$$
\hat{\Gamma}_{0123} \Gamma_{m \bar{n}} \epsilon=i c G_{m \bar{n}} \epsilon
$$

or equivalently

$$
\hat{\Gamma}_{0123 a \bar{b}} \epsilon=i c \delta_{a \bar{b}} \epsilon
$$

where $\delta_{a \bar{b}}$ is the flat space metric with $\delta_{1 \overline{1}}=\frac{1}{2}$ in our conventions 1 . This restriction on $\epsilon$ means that the solution will preserve $\frac{1}{4}$ of the supersymmetry which corresponds to $\mathcal{N}=2$ in four dimensions.

Using these equations we can express the terms on the right hand side of equation (11) in terms of a sum of linearly-independent products of gamma matrices acting on $\epsilon$. The coefficients of these terms must then vanish and this will give us a set of equations which must be satisfied for a solution preserving $\frac{1}{4}$ of the supersymmetry.

It is straightforward to express equation (1) as a sum of independent products of $\Gamma$-matrices and we will only present the final results here. Requiring the coefficient of each term to vanish we get a large number of equations which can be reduced to the following set of independent equations:

$$
\begin{aligned}
H_{1}^{2} & =H_{2}^{-1} \\
H_{2} & =(4 g)^{\frac{2}{3}} \equiv H^{\frac{2}{3}} \\
F_{m \bar{n} \alpha \beta} & =\frac{i c}{2} \epsilon_{\alpha \beta \gamma} \partial_{\gamma} g_{m \bar{n}} \\
F_{m 89(10)} & =-\frac{i c}{2} \partial_{m} H \\
F_{\bar{m} 89(10)} & =\frac{i c}{2} \partial_{\bar{m}} H \\
\partial_{v} g_{s \bar{n}} & =\partial_{s} g_{v \bar{n}} \\
\partial_{\bar{v}} g_{m \bar{s}} & =\partial_{\bar{s}} g_{m \bar{v}}
\end{aligned}
$$

\footnotetext{
${ }^{1}$ We use $a, b$ etc. with values 1 and 2 for the flat space complex coordinates while $m, n$ etc. with values $v$ and $s$ are the curved space complex coordinate indices.
} 
with all other types of components of $F$ vanishing. The last two equations are simply the statement that $g_{m \bar{n}}$ is a Kähler metric. Clearly the full metric and 4-form $F$ are determined explicitly by the Kähler metric.

Now to ensure that the equations of motion of 11-dimensional supergravity are satisfied we only need to satisfy the Bianchi identity and equations of motion for $F$. This will give us the equations to determine $g_{m \bar{n}}$. We note that $F \wedge F=0$ for above $F$. Using the above relations it is easy to verify that the Bianchi identity for the dual 7-form $* F$ :

$$
d * F=0
$$

is automatically satisfied and we have the equations of motion with source term:

$$
d F=J_{m \bar{n}} d z^{m} \wedge d z^{\bar{n}} \wedge d x^{8} \wedge d x^{9} \wedge d x^{10},
$$

where $J$ is the source for $* F$, leading to the following equations:

$$
J_{m \bar{n}}=\frac{i c}{2}\left(4 \partial_{m} \partial_{\bar{n}}(2 g)+\partial_{\gamma} \partial_{\gamma} g_{m \bar{n}}\right)
$$

\section{$2.1 \quad$ Simple example}

We can now check that we reproduce the solution for a single M5-brane with worldvolume 012345. Let

$$
\begin{aligned}
g_{v \bar{v}} & =\frac{1}{2} \\
g_{s \bar{s}} & =\frac{1}{2} H \\
g_{v \bar{s}} & =0 \\
g_{s \bar{v}} & =0
\end{aligned}
$$

Then equations (27) reduce to:

$$
J_{s \bar{s}}=\frac{i c}{4}\left(4 \partial_{s} \partial_{\bar{s}}+\partial_{\gamma} \partial_{\gamma}\right) H
$$

where the source term is just a delta function defining the position of the M5-brane, normalised as:

$$
J_{s \bar{s}}=-i c 2 \pi^{3} l_{p}^{3} \delta^{(3)}(r) \delta^{(2)}(s)
$$


So $\mathrm{H}$ is a solution of the 5-dimensional Laplace equation with a source at the position of the 5-brane. So we find:

$$
H=1+\frac{\pi l_{p}^{3}}{r_{(5)}^{3}}
$$

where $r_{(5)}$ is the radial coordinate in the 5 dimensions transverse to the 5 brane. It is easy to see this reproduces the solution given in [18, 36]. Of course this solution will preserve $\frac{1}{2}$ of the full supersymmetry since it describes only one flat M5-brane.

\subsection{Summary of general solution}

What we have shown is that given a Kähler metric $g_{m \bar{n}}$ on a complex 2dimensional space which satisfies the source equations:

$$
J_{m \bar{n}}=\frac{i c}{2}\left(4 \partial_{m} \partial_{\bar{n}}(2 g)+\partial_{\gamma} \partial_{\gamma} g_{m \bar{n}}\right)
$$

there exists a solution to the equations of motion of 11-dimensional supergravity preserving at least one quarter of the full supersymmetry. The 4-form $F$ is defined explicitly in terms of $g_{m \bar{n}}$ by equations (20)-(22). The metric is given by:

$$
d s^{2}=H^{-\frac{1}{3}} \eta_{\mu \nu} d x^{\mu} d x^{\nu}+2 H^{-\frac{1}{3}} g_{m \bar{n}} d z^{m} d z^{\bar{n}}+H^{\frac{2}{3}} \delta_{\alpha \beta} d x^{\alpha} d x^{\beta}
$$

where:

$$
H=4 g=4\left(g_{v \bar{v}} g_{s \bar{s}}-g_{v \bar{s}} g_{s \bar{v}}\right)
$$

The boundary conditions, equations (8)-(11), for Minkowski space at infinity become simply:

$$
\begin{aligned}
& g_{v \bar{v}} \rightarrow \frac{1}{2} \\
& g_{s \bar{s}} \rightarrow \frac{1}{2} \\
& g_{v \bar{s}} \rightarrow 0 \\
& g_{s \bar{v}} \rightarrow 0
\end{aligned}
$$

We can combine the Kähler conditions and the source equations into equations determining the Kähler potential $K$. The metric is defined as:

$$
g_{m \bar{n}}=\partial_{m} \partial_{\bar{n}} K
$$


and the Kähler potential is determined by:

$$
\frac{i c}{2} \partial_{m} \partial_{\bar{n}}\left(8 g(K)+\partial_{\gamma} \partial_{\gamma} K\right)=J_{m \bar{n}}
$$

These equations are similar to the Monge-Ampere equation and the general solution is not known. It would be possible to find solutions numerically but we will not pursue that approach here. It is also possible to solve asymptotically around flat Minkowski spacetime which would be valid far from the branes. However, we are interested in the near-horizon limit and in the next section we will show the solution in this limit for a special configuration of branes corresponding to the description of conformal QCD.

We note that even without solving equations (43), the general form of the solution exhibits the R-symmetry group appropriate for $\mathcal{N}=2$ YangMills in four dimensions. The overall transverse 3-space clearly has an $\mathrm{SO}(3)$ isometry group and there is also a $\mathrm{U}(1)$ isometry for the superconformal case, since $K$ depends on $v$ only through its absolute value in this case, as we see below. So as expected the global R-symmetry of the gauge theory is manifested as the group of isometries of the metric.

It can be checked that our solution generalises previous solutions for M5branes intersecting orthogonally with a common $(3+1)$-dimensional worldvolume (and related configurations in lower dimensions.) Indeed if we choose $g_{v \bar{s}}=g_{s \bar{v}}=0$ then the Kähler conditions tell us that $g_{v \bar{v}}$ is independent of $s$ and $\bar{s}$ while $g_{s \bar{s}}$ is independent of $v$ and $\bar{v}$. It is then easy to show that equations (27) are equivalent to the curved space Laplace equations in [37. It is important to also keep in mind the allowed coordinate dependence of the functions, imposed in our case by the Kähler condition. This is equivalent to the additional constraints on the 'harmonic' functions, derived in 38. It is easy to see that this ansatz is too restictive to allow solutions for fully localised intersecting branes - at least one of the branes must be smeared in some directions. Our general solution for this type of brane configuration does allow fully localised branes to intersect. The important generalisation seems to be the inclusion of non-diagonal terms in the metric, i.e. we must have non-zero $g_{v \bar{s}}$ and $g_{s \bar{v}}$. The explicit construction of such a solution requires the solution of equations (43) with appropriate fully localised sources. Unfortunately we have so far been unable to find such a solution and this matter is currently under investigation [39]. 


\section{Near-horizon limit of solution in 10 dimen- sions}

We want to consider the case of 2 parallel NS5-branes with worldvolume 012345 and $N$ infinite D4-branes with worldvolume 01236 in type IIA. This is the Hanany-Witten construction of four-dimensional $\mathcal{N}=2 \mathrm{SU}(N)$ gauge theory with $2 N$ hypermultiplets in the fundamental representation. This is a conformal field theory. Since the D4-branes are infinite they can be thought of as aligned D4-branes ending on either side of the NS5-branes. Such a set-up does not result in bending of the branes since the force exerted by a D4-brane on one side of a NS5-brane is exactly compensated by the aligned D4-brane on the other side of the NS5-brane. This fact has the important consequence that when we lift this configuration to M-theory it receives no non-perturbative corrections 2 .

Clearly this 10-dimensional background can be obtained from an 11dimensional background of M5-branes intersecting so as to preserve one quarter supersymmetry, as discussed in section 2. We can lift the HananyWitten configuration to two M5-branes with world-volume 012345 localized at $t=t_{1}, r=0$ and $t=t_{2}, r=0$ respectively; and $N$ M5-branes with world-volume 012367 localized at $v=0, r=0$. The Riemann surface $\Sigma$ mentioned in the previous section is easy to construct using the methods of [17]. According to [17] the Riemann surface can be represented as :

$$
A(v) t^{2}+B(v) t+C(v)=0 \quad\left(\text { with } t=\exp -s / R_{7}\right) .
$$

Since we are compactifying M-theory on a circle of radius $R_{7}$ to give type IIA, we use the single-valued coordinate $t$ instead of $s$.

Now since for arbitrary $t$ we are on the world-volume of the M5-brane only if $v=v_{i}$, i.e. at the locations of the D4-branes, we conclude that $A=\alpha B=\beta C=\prod_{i=1}^{N}\left(v-v_{i}\right)$. The constants $\alpha, \beta$ are fixed by requiring that $t^{2}+t / \alpha+1 / \beta=0$ has solutions at $t=t_{1,2}$, the positions of the two NS5-branes. We will be interested in the case of maximal gauge symmetry

\footnotetext{
${ }^{2}$ To be more precise we mean that there are no corrections to the brane configuration. In field theory one observes 40] that the coupling constant and moduli are in fact redefined compared to their classical values. These redefinitions can be understood as loop and instanton effects. In the brane picture we use the Seiberg-Witten curve to determine the geometry. However, as observed in [40] the curve itself does not change; only the precise relationship between microscopic and low-energy quantities is modified. So in this sense there are no non-perturbative effects.
} 
enhancement when the D4-branes coincide, i.e. $v_{i}=0$. The Riemann surface $\Sigma$ then splits up into three holomorphic cycles: i) $v=0, t$ arbitrary, ii) $t=t_{1}$, $v$ arbitrary, iii) $t=t_{2}$ with $v$ arbitrary. Thus the lift of the type IIA HananyWitten configuration is 2 M5-branes localized at $t=t_{1,2}$ and $N$ M5-branes localized at $v=0$ with the overall transverse coordinates $x_{\alpha}=0$. In the following section we will show in detail how to use these results to construct the 10-dimensional metric.

Since we wish to make contact with Maldacena's conjecture, we will really be interested in the near-horizon limit of this solution. Here we use a naive interpretation of near-horizon as simply meaning that the harmonic functions approach zero at infinity instead of 1 . This limit will in fact allow us to find a solution where the $N$ D4-branes are fully localised. We will then show that in the appropriate limit the metric contains an $A d S_{5}$ factor as is required for the equivalence via Maldacena's duality to a 4-dimensional conformal field theory.

\subsection{1 to 10 dimensions}

We have 2 M5-branes with worldvolume 012345 and $N$ with worldvolume 012367. We will compactify the 7 direction on a circle to produce the required Hanany-Witten brane construction in type IIA. For simplicity we will assume translation invariance in the 6 direction as well as the 7 direction, i.e. we will have the $N$ M5-branes fully localised but the 2 M5-branes will be 'smeared' in the 6 and 7 directions (or $s$ and $\bar{s}$ directions.) The standard relation between 11-dimensional supergravity and 10-dimensional type IIA 'stringframe' metrics is 41, 42]:

$$
d s_{11}^{2}=e^{-\frac{2 \phi}{3}} d s_{10}^{2}+e^{\frac{4 \phi}{3}}\left(d x_{7}+A_{i} d x^{i}\right)^{2}
$$

Using the form of the 11-dimensional solution, equation (36), we find:

$$
\begin{aligned}
e^{\frac{4 \phi}{3}} & =2 H^{-\frac{1}{3}} g_{s \bar{s}} \\
A_{4} & =\frac{-i}{2 g_{s \bar{s}}}\left(g_{v \bar{s}}-g_{s \bar{v}}\right) \\
A_{5} & =\frac{1}{2 g_{s \bar{s}}}\left(g_{v \bar{s}}+g_{s \bar{v}}\right)
\end{aligned}
$$


with all other components of $A_{i}$ vanishing. So the general form of the 10 dimensional metric is:

$$
\begin{aligned}
d s_{10}^{2}= & \frac{1}{\sqrt{2}} g^{-\frac{1}{2}} g_{s \bar{s}}^{\frac{1}{2}} \eta_{\mu \nu} d x^{\mu} d x^{\nu}+\sqrt{2} g^{\frac{1}{2}} g_{s \bar{s}}^{-\frac{1}{2}} d v d \bar{v}+2 \sqrt{2} g^{\frac{1}{2}} g_{s \bar{s}}^{\frac{1}{2}} \delta_{\alpha \beta} d x^{\alpha} d x^{\beta}+ \\
& \sqrt{2} g^{-\frac{1}{2}} g_{s \bar{s}}^{\frac{3}{2}}\left(d x_{6}+\frac{1}{2 g_{s \bar{s}}}\left(g_{s \bar{v}}+g_{v \bar{s}}\right) d x_{5}+\frac{i}{2 g_{s \bar{s}}}\left(g_{s \bar{v}}-g_{v \bar{s}}\right) d x_{4}\right)^{2}
\end{aligned}
$$

The 10-dimensional 3- and 4-form field strengths are given in terms of the 11-dimensional 4-form by 42]:

$$
\begin{aligned}
F_{M N P Q}^{10-d} & =F_{M N P Q}^{11-d} \\
H_{M N P} & =2 F_{M N P 7}^{11-d}
\end{aligned}
$$

\subsection{Ansatz for solution}

We will now assume that the metric $g_{m \bar{n}}$ is of the form:

$$
\begin{aligned}
g_{s \bar{s}} & =\frac{q_{1}}{r} \\
g_{v \bar{v}} & =g_{v \bar{v}}(|v|, r) \\
g_{s \bar{v}} & =g_{v \bar{s}}=0
\end{aligned}
$$

where $r=\sqrt{\delta_{\alpha \beta} x^{\alpha} x^{\beta}}$ is the radial coordinate in the overall transverse 3dimensional space and $q_{1}$ is a constant. Note that this ansatz guarantees that $g_{m \bar{n}}$ is Kähler.

So now we must find a solution to the source equations (27). The sources will be a delta function in the 3 overall transverse dimensions corresponding to the 2 smeared 5-branes and a delta function in the 5-dimensional space transverse to the $N 5$-branes. So we see that equations (27) are satisfied, up to normalisation of $q_{1}$, by our ansatz except for the case of the $v \bar{v}$ components. For the $v \bar{v}$ components we have (using a coordinate transformation used in 21]):

$$
\begin{aligned}
8 \partial_{v} \partial_{\bar{v}} g+\partial_{\gamma} \partial_{\gamma} g_{v \bar{v}} & =\frac{2 q_{1}}{r} \frac{1}{|v|} \partial_{|v|}\left(|v| \partial_{|v|} g_{v \bar{v}}\right)+\frac{1}{r^{2}} \partial_{r}\left(r^{2} \partial_{r} g_{v \bar{v}}\right) \\
& =\frac{16 q_{1}^{2}}{\rho^{2}} \frac{1}{|v|} \partial_{|v|}\left(|v| \partial_{|v|} g_{v \bar{v}}\right)+\frac{16 q_{1}^{2}}{\rho^{5}} \partial_{\rho}\left(\rho^{3} \partial_{\rho} g_{v \bar{v}}\right) \\
& =\frac{16 q_{1}^{2}}{\rho^{2}} \frac{1}{R^{5}} \partial_{R}\left(R^{5} \partial_{R} g_{v \bar{v}}\right)
\end{aligned}
$$


where $\rho=\sqrt{8 q_{1} r}$ and $R^{2}=\rho^{2}+|v|^{2}$ and in the last step we have assumed that $g_{v \bar{v}}$ is only a function of $R$.

So we can now see that we have a solution for $g_{v \bar{v}}$ :

$$
g_{v \bar{v}}=\frac{q_{2}}{R^{4}}=\frac{q_{2}}{\left(8 q_{1} r+|v|^{2}\right)^{2}}
$$

where $q_{2}$ is a constant. We will now fix the constants $q_{1}$ and $q_{2}$.

In M-theory the normalization of the source equations is as follows:

$$
\begin{aligned}
\nabla^{2} g_{s \bar{s}}+8 \partial_{s} \partial_{\bar{s}} g & =-4 \pi^{3} l_{p}^{3} \delta^{(3)}(r)\left(\delta^{(2)}(s-L / 2)+\delta^{(2)}(s+L / 2)\right) \\
\nabla^{2} g_{v \bar{v}}+8 \partial_{v} \partial_{\bar{v}} g & =-N 4 \pi^{3} l_{p}^{3} \delta^{(3)}(r) \delta^{(2)}(v) \\
\nabla^{2} g_{v \bar{s}}+8 \partial_{v} \partial_{\bar{s}} g & =0
\end{aligned}
$$

We can reduce the solution down to 10-dimensions by compactifying $x_{7}$ with radius $R_{7}$. When $R_{7}$ is small enough we can smear branes in the $x_{7}$ direction and replace the delta function by $1 / 2 \pi R_{7}$. U. Using the relation $l_{p}^{3}=R_{7} \alpha^{\prime}=$ $g_{s}\left(\alpha^{\prime}\right)^{3 / 2}$ we get:

$$
\begin{aligned}
\nabla^{2} g_{s \bar{s}}+2 \partial_{6} \partial_{6} g & =-2 \pi^{2} \alpha^{\prime} \delta^{(3)}(r)\left(\delta\left(x_{6}-L / 2\right)+\delta\left(x_{6}+L / 2\right)\right) \\
\nabla^{2} g_{v \bar{v}}+8 \partial_{v} \partial_{\bar{v}} g & =-N 4 \pi^{3} g_{s}\left(\alpha^{\prime}\right)^{3 / 2} \delta^{(3)}(r) \delta^{(2)}(v) \\
\nabla^{2} g_{v \bar{s}}+4 \partial_{v} \partial_{6} g & =0 .
\end{aligned}
$$

Finally, we smear the 2 NS5-branes in the $x_{6}$ direction. We compactify the $x_{6}$ direction with radius $\sqrt{\alpha^{\prime}} / c_{1}$. Smearing in the 6 direction, we can replace the delta functions by $c_{1} / 2 \pi \sqrt{\alpha^{\prime}}$. More generally, if we have $n$ NS5-branes the source equation becomes:

$$
\nabla^{2} g_{s \bar{s}}+2 \partial_{6} \partial_{6} g=-\pi n c_{1}\left(\alpha^{\prime}\right)^{1 / 2} \delta^{(3)}(r) .
$$

We can now fix the normalization

$$
q_{1}=n\left(\alpha^{\prime}\right)^{1 / 2} c_{1} / 4
$$

and after inserting appropriate volume factors to convert the 6-dimensional delta function at $R=0$ in equation (55) to delta functions in $r$ and $v$ :

$$
\begin{aligned}
q_{2} & =8 \pi g_{s}\left(\alpha^{\prime}\right)^{3 / 2} N q_{1} \\
& =2 \pi g_{s}\left(\alpha^{\prime}\right)^{2} n N c_{1}
\end{aligned}
$$

\footnotetext{
${ }^{3}$ This procedure gives the same result as the Poisson summation of the harmonic function given in 443.
} 


\subsection{Decoupling limit}

The near-horizon limit is one in which field theory quantities are held fixed while decoupling the bulk degrees of freedom. It is natural to express the metric in terms of variables appropriate to this limit.

In this system of D4-branes and NS5-branes we would like to preserve the masses of field theory excitations and the field theory coupling constant. The moduli space of the field theory is parameterized by a complex adjoint Higgs field. On the supergravity side the Higgs expectation values are given by the positions of the D4-branes in $v$. The mass of a string stretched between two D4-branes is $|w|=|v| / \alpha^{\prime}$ where $|v|$ is the coordinate distance between the branes. These states are "electrically" charged. There are also magnetically charged states which are described by D2-branes stretched between D4-branes. They have mass:

$$
m=\frac{|v| L}{g_{s}\left(\alpha^{\prime}\right)^{3 / 2}}=\frac{|v|}{g_{Y M}^{2} \alpha^{\prime}}=\frac{|w|}{g_{Y M}^{2}},
$$

where $L$ is the coordinate distance between the two NS5-branes. Thus in the $\alpha^{\prime} \rightarrow 0$ limit if we keep $w=v / \alpha^{\prime}$ and the Yang-Mills coupling constant $g_{Y M}$ fixed, the field theory states have finite mass.

The field theory living on the D4-branes has only $v$ as a modulus and the field theory excitations only determine the scaling of $v$. The remaining coordinates $r, x_{6}$ in the decoupling limit have their scalings determined by keeping excitations on the NS5-brane and the Yang-Mills coupling constant fixed. The NS5-branes have D2-branes ending on them. The ends of the D2brane appear as strings in the NS5-brane's world volume theory with tension $r / g_{s} \alpha^{\prime 3 / 2}$. In the decoupling limit we take $\alpha^{\prime} \rightarrow 0$ while keeping $r / g_{s} \alpha^{\prime 3 / 2}$ fixed.

Lastly the scaling of $x_{6}$ can be determined as follows in the decoupling limit. The Yang-Mills coupling constant is given by $g_{Y M}^{-2}=L / g_{s}\left(\alpha^{\prime}\right)^{1 / 2}$, where $L$ is the coordinate distance between the two NS5-branes. Thus to keep the coupling constant fixed in the field theory we have to take the limit $\alpha^{\prime} \rightarrow 0$ while keeping $x_{6} / g_{s} \alpha^{1 / 2}$ fixed.

With $n$ NS5-branes the gauge group is $\mathrm{SU}(N)^{n}$. The gauge coupling of each $\mathrm{SU}(N)$ group is given by the separation, $L$, of two consecutive NS5branes in the $x_{6}$ direction. Since we have smeared the NS5-branes around the circle, they should be considered to be equally spaced. This circle has 
radius $\sqrt{\alpha^{\prime}} / c_{1}$ so $L=2 \pi \sqrt{\alpha^{\prime}} /\left(n c_{1}\right)$. So we have:

$$
\frac{1}{g_{Y M}^{2}}=\frac{L}{g_{s} \sqrt{\alpha^{\prime}}}=\frac{2 \pi}{n g_{s} c_{1}}
$$

So we should keep $n g_{s} c_{1}$ fixed or if we take the large $N$ limit with fixed 't Hooft coupling, we should keep $n N g_{s} c_{1}$ fixed.

So we will define variables $u, w$ and $t$ with dimensions of mass and a dimensionless angular variable $\Phi$ :

$$
\begin{aligned}
u & =\frac{R}{\alpha^{\prime}} \\
w & =\frac{v}{\alpha^{\prime}} \\
t^{2} & =\frac{r}{g_{s} \alpha^{\frac{3}{2}}} \\
\Phi & =\frac{c_{1} x_{6}}{\sqrt{\alpha^{\prime}}}
\end{aligned}
$$

In terms of these variables the non-zero components of the complex metric are:

$$
\begin{aligned}
g_{v \bar{v}} & =\frac{2 \pi g_{s} n N c_{1}}{\left(\alpha^{\prime}\right)^{2} u^{4}} \\
g_{s \bar{s}} & =\frac{n c_{1}}{4 g_{s} \alpha^{\prime} t^{2}}
\end{aligned}
$$

Then we can express the 10-dimensional metric in terms of these variables as:

$$
\begin{aligned}
\frac{1}{\alpha^{\prime}} d s_{10}^{2}= & u^{2} Q^{-\frac{1}{2}} \eta_{\mu \nu} d x^{\mu} d x^{\nu}+u^{-2} Q^{\frac{1}{2}} d w d \bar{w}+ \\
& u^{2} Q^{-\frac{1}{2}} \frac{n c_{1}}{2 \alpha^{\prime} c_{1} t^{2}} d \Phi^{2}+u^{-2} Q^{\frac{1}{2}} \frac{n g_{s} c_{1}}{2}\left(4 d t^{2}+t^{2} d \Omega_{2}^{2}\right)
\end{aligned}
$$

where we have defined:

$$
Q=4 \pi g_{s} n N c_{1}
$$

We now define the dimensionless variables $\eta$ and $\theta$ with the ranges $0 \leq \eta \leq \frac{\pi}{2}$ and $0 \leq \theta<2 \pi$ :

$$
\begin{aligned}
w & =|w| e^{i \theta} \\
|w| & =u \sin \eta
\end{aligned}
$$


Then we have:

$$
u^{2} \cos ^{2} \eta=2 n c_{1} g_{s} t^{2} .
$$

We also write $d \Omega_{2}^{2}$ in terms of angular variables $0 \leq \omega \leq \pi$ and $0 \leq \sigma<2 \pi$ as:

$$
d \Omega_{2}^{2}=d \omega^{2}+\sin ^{2} \omega d \sigma^{2}
$$

We can now rewrite the metric in a form where the $A d S_{5}$ factor is explicit:

$$
\begin{aligned}
\frac{1}{\alpha^{\prime}} d s_{10}^{2}= & \left(\frac{u^{2}}{\sqrt{Q}} \eta_{\mu \nu} d x^{\mu} d x^{\nu}+\frac{\sqrt{Q}}{u^{2}} d u^{2}\right)+\left(\sqrt{Q} d \eta^{2}+\sqrt{Q} \sin ^{2} \eta d \theta^{2}+\right. \\
& \left.\frac{n^{2}}{\sqrt{Q} \cos ^{2} \eta} d \Phi^{2}+\frac{\sqrt{Q}}{4} \cos ^{2} \eta\left(d \omega^{2}+\sin ^{2} \omega d \sigma^{2}\right)\right)
\end{aligned}
$$

It is straightforward to find the non-zero 3- and 4-form field strengths. They are given by:

$$
\begin{aligned}
& H_{\Phi \omega \sigma}=-\frac{c}{2} n \alpha^{\prime} \sin \omega \\
& F_{\eta \theta \omega \sigma}=-2 \pi c g_{s} N\left(\alpha^{\prime}\right)^{3 / 2} \sin \eta \cos ^{3} \eta \sin \omega
\end{aligned}
$$

Since $H=d B$ we can calculate the non-trivial part of the B-field. We find:

$$
B_{\Phi \sigma}=-\frac{c}{2} n \alpha^{\prime}\left(\cos \omega+c_{0}\right)
$$

where we have chosen to keep the constant of integration $c_{0}$.

\section{T-Duality}

Using the T-duality rules [44, 45, 42] we can T-dualise the metric (77) to a type IIB metric for D3-branes at an $A_{n-1}$ singularity. The near horizon limit is described by type IIB supergravity on the space $A d S_{5} \times\left(S^{5} / \mathbf{Z}_{\mathbf{n}}\right)$. The AdS/CFT correspondence in this case has been discussed in [4, 5]. We perform the T-duality in the 6 direction which we have already considered to be compact, parameterised by the angular coordinate $\Phi$. As described in [24], T-duality transverse to $n$ NS5-branes transforms them to $n$ KaluzaKlein 5-branes which are equivalent to an $n$-centred Taub-NUT space in the transverse directions. When the centres coincide as in this case there is an $A_{n-1}$ singularity and at low energies we can simply describe the space as a 
$\mathbb{C}^{2} / \mathbf{Z}_{\mathbf{n}}$ orbifold. In our case where the D4-branes fill the 6 direction we will end up with D3-branes at the orbifold fixed point. However this T-duality can still be performed for general Hanany-Witten setups with D4-branes ending on NS5-branes. In this case the D4-branes will T-dualise to fractional D3-branes at the orbifold fixed point 27, 28]. So the problem of finding the supergravity solution for a general (non-conformal) Hanany-Witten setup is equivalent to finding the metric for fractional D3-branes at an orbifold fixed point.

We now briefly describe how to write down the metric for D3-branes in the presence of an $A_{k-1}$ ALE singularity. An ALE space with an $A_{k-1}$ singularity can be defined as a $\mathbb{C}^{2} / \mathbf{Z}_{\mathbf{k}}$ orbifold, where the action of the $\mathbf{Z}_{\mathbf{k}}$ is given as follows:

$$
\begin{aligned}
& z_{1} \rightarrow \exp \left(i \frac{2 \pi}{k}\right) z_{1} \\
& z_{2} \rightarrow \exp \left(-i \frac{2 \pi}{k}\right) z_{2} .
\end{aligned}
$$

The coordinates $z_{1}, z_{2}$ are covering space coordinates of the ALE space, and in these coordinates the metric of the ALE space is flat. Thus in the covering space coordinates one can easily write down the solution for D3-branes [7, 9]. In fact, it is identical to the usual metric [46] except now one has to introduce images of the D3-branes due to the identification (81). To have $N$ D3-branes in the presence of the ALE singularity one has to introduce $N k$ D3-branes in the covering space, where $N(k-1)$ of them are images. The metric for all $N$ D3-branes localized at the origin is given by:

$$
d s^{2}=f^{-1 / 2} d x_{\|}^{2}+f^{1 / 2}\left(|d v|^{2}+\left|d z_{1}\right|^{2}+\left|d z_{2}\right|^{2}\right)
$$

where

$$
f=1+\frac{4 \pi g_{s} k N \alpha^{2}}{r^{4}}
$$

We can now change variables from the covering space to single-valued variables as in [21]:

$$
\begin{aligned}
\rho^{2} & =\left|z_{1}\right|^{2}+\left|z_{2}\right|^{2} \\
z_{1} & =\rho \cos \frac{\theta}{2} e^{i \frac{\delta}{k}} \\
z_{2} & =\rho \sin \frac{\theta}{2} e^{i \eta-i \frac{\delta}{k}}
\end{aligned}
$$


with $0 \leq \theta \leq \pi, 0 \leq \delta<2 \pi$ and $0 \leq \eta<2 \pi$. We also introduce

$$
\begin{aligned}
& \rho=r \sin \gamma \\
& v=r \cos \gamma e^{i \phi}
\end{aligned}
$$

with $0 \leq \gamma \leq \pi / 2,0 \leq \phi<2 \pi$. We can now take the near-horizon limit $\alpha^{\prime} \rightarrow 0$ with $u=r / \alpha^{\prime}$ held fixed where we can ignore the constant term in $f$ and write (82) as:

$$
\begin{aligned}
\frac{1}{\alpha^{\prime}} d s_{10}^{2}= & \left(\frac{u^{2}}{\sqrt{Q}} \eta_{\mu \nu} d x^{\mu} d x^{\nu}+\frac{\sqrt{Q}}{u^{2}} d u^{2}\right)+\sqrt{Q}\left(d \gamma^{2}+\cos ^{2} \gamma d \phi^{2}+\right. \\
& \frac{1}{4} \sin ^{2} \gamma d \theta^{2}+\frac{1}{4} \sin ^{2} \gamma \sin ^{2} \theta d \eta^{2}+ \\
& \left.\frac{1}{k^{2}} \sin ^{2} \gamma\left(d \delta-\frac{k}{2}(\cos \theta-1) d \eta\right)^{2}\right) .
\end{aligned}
$$

where $Q$ is defined in terms of the IIB string coupling by:

$$
Q=4 \pi g_{s} k N .
$$

We will now show that the T-dual of our IIA metric is precisely this metric, with $k$ identified with $n$, the number of NS5-branes. This provides a nontrivial check of our solution and normalisations.

For the solution (77) the T-duality rules are fairly simple since the metric is diagonal and only a few gauge fields are non-trivial. From [44, 45, 42] we have the following transformation rules for T-duality along the $\Phi$ direction:

$$
\begin{aligned}
g_{\Phi \Phi} & =g_{\Phi \Phi}^{-1} \\
g_{\Phi \sigma} & =B_{\Phi \sigma} g_{\Phi \Phi}^{-1} \\
g_{\sigma \sigma} & =g_{\sigma \sigma}+\left(B_{\Phi \sigma}\right)^{2} g_{\Phi \Phi}^{-1} \\
g_{M N} & =g_{M N} \\
\exp (\phi) & =\exp (\phi) g_{\Phi \Phi}^{-\frac{1}{2}} \\
F_{\Phi \eta \theta \omega \sigma}^{(5)} & =-\frac{3}{10} F_{\eta \theta \omega \sigma}
\end{aligned}
$$

where all quantities on the left are the type IIB parameters, determined by the type IIA parameters on the right. The indices $M, N$ are any spacetime indices except $\Phi$ and $\sigma . \quad F^{(5)}$ is the 5-form field strength in type IIB and 
self-duality must be imposed. The transformations are written for $\alpha^{\prime}=1$. It is now a simple matter to apply the T-duality rules and we find the type IIB metric:

$$
\begin{aligned}
\frac{1}{\alpha^{\prime}} d s_{10}^{2}= & \left(\frac{u^{2}}{\sqrt{Q}} \eta_{\mu \nu} d x^{\mu} d x^{\nu}+\frac{\sqrt{Q}}{u^{2}} d u^{2}\right)+\left(\sqrt{Q} d \eta^{2}+\sqrt{Q} \sin ^{2} \eta d \theta^{2}+\right. \\
& \frac{\sqrt{Q}}{4} \cos ^{2} \eta d \omega^{2}+\frac{\sqrt{Q}}{4} \cos ^{2} \eta \sin ^{2} \omega d \sigma^{2}+ \\
& \left.\frac{\sqrt{Q} \cos ^{2} \eta}{n^{2}}\left(d \Phi-c \frac{n}{2}\left(\cos \omega+c_{0}\right) d \sigma\right)^{2}\right)
\end{aligned}
$$

The IIB string coupling is given in terms of the IIA string coupling by equation (93). Our IIA metric is only valid in the near-horizon limit so we can't substitute $g_{\Phi \Phi}$ into equation (93) to relate the string couplings which are defined asymptotically. However we have normalised our solution so that the metric should be asymptotically flat. So asymptotically we must have $g_{\Phi \Phi}=1 / c_{1}^{2}$ since the radius of the $\Phi$ direction is $\sqrt{\alpha^{\prime}} / c_{1}$. So the string couplings are related by:

$$
g_{s}^{I I B}=c_{1} g_{s}^{I I A}
$$

and so in terms of IIB quantities we have:

$$
Q=4 \pi g_{s} n N
$$

So we see that with $c_{0}=-1$ we reproduce the metric for $n$ D3-branes at a $\mathbb{C}^{2} / \mathbf{Z}_{\mathbf{n}}$ orbifold fixed point. The relation between the variables in equation (87) and our notation is easily seen to be: $\sin \eta=\cos \gamma, \theta=\phi, \omega=\theta$, $c \sigma=\eta$ and $\Phi=\delta$.

Finally we give the non-trivial type IIB 5 -form components:

$$
(* F)_{\Phi \eta \theta \omega \sigma}=F_{\Phi \eta \theta \omega \sigma}=\frac{3 \pi c}{5 c_{1}} g_{s} N\left(\alpha^{\prime}\right)^{2} \sin \eta \cos ^{3} \eta \sin \omega
$$

\section{Conclusions}

We have shown the general form of the 11-dimensional supergravity solution describing a 5-brane configuration with worldvolume $\mathbb{R}^{4} \times \Sigma$ with $\Sigma$ being a holomorphic 2-cycle in $\mathbb{C}^{2}$. This corresponds to a single 5 -brane or multiple 5 -branes depending on the form of $\Sigma$, i.e. whether or not $\Sigma$ is simply connected. We have also shown the reduction of this solution to 10 dimensions 
by compactifying one real coordinate of $\mathbb{C}^{2}$ on a circle. This configuration will generically correspond to a system of D4- and NS5-branes in type IIA string theory.

We were unable to fully solve the source equations (27) but in the special case we were interested in we could find a near-horizon solution for $\mathrm{N}$ fully localised 5-branes intersecting n 5-branes which were smeared along the localised 5-branes. Apart from the smearing, this reduces to a Hanany-Witten configuration in type IIA describing the $\mathcal{N}=2$ conformal field theory with gauge group $\mathrm{SU}(N)^{n}$. Taking the near-horizon limit in supergravity should give the Maldacena dual of the CFT and indeed we see that this is plausible since we showed that the space factorises into the form $A d S_{5} \times M$ where $M$ is a compact manifold with the isometries of the field theory R-symmetry group in addition to a $\mathrm{U}(1)$ due to smearing.

There are a number of directions one can pursue. One is to construct the M-theory solution for non-conformal field theories in which the corresponding type IIA picture has non-aligned D4-branes. Of interest also in the conformal theories considered here is to fully localise the NS5-branes. This would allow arbitrary gauge couplings for each $\mathrm{SU}(N)$ factor and would permit solutions with finite gauge couplings without compactifying $x_{6}$. From the supergravity point of view it would also be interesting to fully localise the M5-branes in 11 dimensions. Another question [39] which one can address is that of conformal field theories with more exotic matter content than fundamental and adjoint matter. For example by introducing a third NS5-brane and an orientifold 6-plane 47] one can have symmetric or anti-symmetric second rank tensor representations.

\section{Acknowledgements}

We would like to thank J. Maldacena, Ö. Sarioglu, D. Sorokin and C. Vafa for very helpful discussions. AF is supported by NSF grant PHY-93-15811. DS is supported by the Deutsche Forschungs Gemeinschaft.

\section{Note added}

A paper by D. Youm 48 appeared in the archives on the same day as this paper and another paper by A. Loewy 49] appeared shortly after. Both these papers have some overlap with our results in section 3 . 


\section{References}

[1] J.M. Maldacena The Large $N$ Limit of Superconformal Field Theories and Supergravity Adv. Theor. Math. Phys. 2 (1998) 231-252; hepth/9711200.

[2] S. Gubser, I. Klebanov and A. Polyakov Gauge theory correlators from noncritical string theory Phys. Lett. B428 (1998) 105; hep-th/9802109.

[3] E. Witten Anti-de Sitter space and holography Adv. Theor. Math. Phys. 2 (1998) 253; hep-th/9802150.

[4] S. Kachru and E. Silverstein 4-D conformal theories and strings on orbifolds Phys. Rev. Lett. 80 (1998) 4855; hep-th/9802183.

[5] A. Lawrence, N. Nekrasov and C. Vafa On Conformal Theories in Four Dimensions Nucl. Phys. B533 (1998) 199; hep-th/9803015.

[6] M. Bershadsky, Z. Kakushadze and C. Vafa String Expansion as Large $N$ Expansion of Gauge Theories Nucl. Phys. B523 (1998) 59; hepth/9803076.

[7] A. Fayyazuddin and M. Spalinski Large N Superconformal Gauge Theories and Supergravity Orientifolds Nucl. Phys. B535 (1998) 219; hepth/9805096.

[8] A. Kehagias New type IIB vacua and their F theory interpretation Phys. Lett. B435 (1998) 337 hep-th/9805131.

[9] O. Aharony, A. Fayyazuddin and J. Maldacena The Large $N$ limit of $\mathcal{N}=2,1$ Field Theories from Threebranes in F-theory JHEP 9807 (1998) 013; hep-th/9806159.

[10] I.R. Klebanov and E. Witten Superconformal field theory on threebranes at a Calabi-Yau singularity Nucl. Phys. B536 (1998) 199; hepth/9807080.

[11] S.S. Gubser Einstein manifolds and conformal field theories Phys. Rev. D59 (1999) 025006; hep-th/9807164.

[12] E. Lopez A Family of $N=1 S U(N)^{k}$ theories from branes at singularities hep-th/9812025. 
[13] A. Khavaev, K. Pilch and N.P. Warner New Vacua of Gauged N=8 Supergravity hep-th/9812035.

[14] A. Karch, D. Lust and A. Miemiec New Superconformal Field Theories and their Supergravity Description hep-th/9901041.

[15] I.R. Klebanov and A.A. Tseytlin A Non-supersymmetric Large N CFT from Type 0 String Theory hep-th/9901101.

[16] A. Hanany and E. Witten Type IIB Superstrings, BPS Monopoles, And Three-Dimensional Gauge Dynamics Nucl. Phys. B492 (1997) 152-190; hep-th/9611230.

[17] E. Witten Solutions Of Four-Dimensional Gauge Theories Via M Theory Nucl. Phys. B500 (1997) 3-42; hep-th/9703166.

[18] J. Gauntlett Intersecting Branes hep-th/9705011.

[19] H. Lu, C.N. Pope, T.A. Tran and K.W. Xu Classification of p-branes, NUTs, waves and intersections Nucl. Phys. B511 (1997) 98; hepth/9708055.

[20] K.S. Stelle BPS branes in supergravity hep-th/9803116.

[21] N. Itzhaki, A.A. Tseytlin and S. Yankielowicz Supergravity solutions for branes localized within branes Phys. Lett. B432 (1998) 298; hepth/9803103.

[22] A. Hashimoto Supergravity solutions for localized intersections of branes hep-th/9812159.

[23] H. Yang Localized intersecting brane solutions of $D=11$ supergravity hep-th/9902128.

[24] H. Ooguri and C. Vafa Two-dimensional black hole and singularities of CY manifolds Nucl. Phys. B463 (1996) 55; hep-th/9511164.

[25] E. Witten Some comments on string dynamics hep-th/9507121.

[26] A. Klemm, W. Lerche, P. Mayr, C. Vafa and N. Warner Selfdual strings and N=2 supersymmetric field theory Nucl. Phys. B477 (1996) 746; hep-th/9604034. 
[27] A. Karch, D. Lust and D.J. Smith Equivalence of geometric engineering and Hanany-Witten via fractional branes Nucl. Phys. B533 (1998) 348; hep-th/9803232.

[28] D.J. Smith Equivalence of geometric engineering and Hanany-Witten Proceedings of the 32nd International Symposium Ahrenshoop on the Theory of Elementary Particles, Buckow, Germany, 1-5 September 1998; Proceedings of the 2nd Conference on Quantum Aspects of Gauge Theories, Supersymmetry and Unification, Corfu, Greece, 21-26 September 1998; hep-th/9812126.

[29] A.M. Uranga Brane configurations for branes at conifolds hepth/9811004.

[30] K. Dasgupta and S. Mukhi Brane constructions, conifolds and M theory hep-th/9811139.

[31] R. von Unge Branes at generalized conifolds and toric geometry hepth/9901091.

[32] K. Becker, M. Becker and A. Strominger Five-branes, membranes and nonperturbative string theory Nucl. Phys. B456 (1995) 130; hepth/9507158.

[33] A. Fayyazuddin and M. Spalinski The Seiberg-Witten differential from $M$ theory Nucl. Phys. B508 (1997) 219; hep-th/9706087.

[34] M. Cvetic and C.M. Hull Wrapped branes and supersymmetry Nucl. Phys. B519 (1997) 141; hep-th/9709033.

[35] A. Fayyazuddin and M. Spalinski Extended objects in $M Q C D$ hepth/9711083.

[36] R. Güven Black p-brane solutions of $D=11$ supergravity theory Phys. Lett. B276 (1991) 49.

[37] A.A. Tseytlin Harmonic superpositions of M-branes Nucl. Phys. B475 (1996) 149; hep-th/9604035.

[38] H. Lu and C.N. Pope Interacting intersections Int. J. Mod. Phys. A13 (1998) 4425; hep-th/9710155. 
[39] A. Fayyazuddin and D.J. Smith Work in progress

[40] N. Dorey, V.V. Khoze and M.P. Mattis On N=2 supersymmetric QCD with four flavors Nucl. Phys. B492 (1997) 607; hep-th/9611016.

[41] E. Witten String theory dynamics in various dimensions Nucl. Phys. B443 (1995) 85; hep-th/9503124.

[42] E. Bergshoeff, C. Hull and T. Ortin Duality in the type II superstring effective action Nucl. Phys. B451 (1995) 547; hep-th/9504081.

[43] N. Itzhaki, J. M. Maldacena, J. Sonnenschein and S. Yankelowicz Supergravity and The Large $N$ Limit of Theories With Sixteen Supercharges hep-th/9802042.

[44] T.H. Buscher A symmetry of the string background field equations Phys. Lett. B194 (1987) 59.

[45] T.H. Buscher Path-integral derivation of quantum duality in nonlinear sigma-models Phys. Lett. B201 (1988) 466.

[46] G. Horowitz and A. Strominger Black strings and p-Branes Nucl. Phys. B360 (1991) 197.

[47] K. Landsteiner, E. Lopez and D.A. Lowe Supersymmetric gauge theories from branes and orientifold six planes JHEP 07 (1998) 011; hepth/9805158.

[48] D. Youm Localized intersecting BPS branes hep-th/9902208.

[49] A. Loewy Semilocalized brane intersections in SUGRA hep-th/9903038. 